\title{
A Rare Cause of Gross Hematuria in Childhood: Renal Lymphangiectasia
}

\author{
๑ Çağla Serpil Doğan, @ Gülşah Kaya Aksoy
}

University of Health Sciences, Antalya Training and Research Hospital, Clinic of Pediatrics, Division of Pediatric Nephrology, Antalya, Turkey

\begin{abstract}
Renal lymphangiectasia is a rare disorder characterized by cystic malformation of the lymphatic ducts surrounding the kidney. In this article, an adolescent who presented with painless gross hematuria, hypertension, polycythemia and who was determined to have left perirenal lymphangiectasia and ipsilateral decreased renal function on imaging studies is reported.

Keywords: Renal lymphangiectasia, child, hematuria
\end{abstract}

\section{Introduction}

Renal lymphangiectasia ( $\mathrm{RL}$ ) is a rare benign malformation of the lymphatic system surrounding the kidney. It is characterized by a cystic dilatation of the peripelvic (intrarenal) and/or perirenal lymphatic ducts due to a miscommunication between the renal - perirenal lymphatic ducts and the retroperitoneal lymphatic trunks. It is bilateral in more than $90 \%$ of cases. The etiology of this disorder remains unknown, nevertheless, familial, developmental and acquired causes have been presumed $(1,2)$.

$\mathrm{RL}$ is incidentally detected in most cases, however, it may have non-specific presentation such as abdominal pain, flank pain, hematuria and, rarely lymphedema, ascites or pleural effusion. Although benign, it may be complicated by hypertension, polycythemia, chronic renal failure or renal vein thrombosis (3-6).

Herein, we report a pediatric case who presented with gross hematuria, hypertension, polycythemia and ipsilateral decreased renal function probably linked to local compression of unilateral perirenal lymphangiectasia.

\section{Case Report}

A 13 year-old boy was referred to our clinic with painless gross hematuria which had been present for the previous 2 days without any other symptoms. He had no remarkable medical or family history. There was no history of trauma. Physical examination was normal except for mild hypertension. His blood pressure was 130/90 mmHg and was above the $95^{\text {th }}$ percentile value for age, sex and height. Twenty-four-hour ambulatory blood pressure (ABP) monitoring was also performed and mean systolic and diastolic BP values were found $>95^{\text {th }}$ percentile of the $A B P$ norm (stage 1 hypertension). A complete blood count showed polycythemia, with red blood cell (RBC): $5.7 \times 10^{6} / \mathrm{mm}^{3}(4.1-5.2$ x10 $/ \mathrm{mm}^{3}$ ), hemoglobin: 17.2g/dL (12.8-15.0 g/dL), hematocrit: 51\% (37.3-47.3\%), mean corpuscular volume: 90 fL (81.4-91.9

Address for Correspondence

Çağla Serpil Doğan MD, University of Health Sciences, Antalya Training and Research Hospital, Clinic of Pediatrics, Division of Pediatric Nephrology, Antalya, Turkey Phone: +90 5325842951 E-mail: d.caglaserpil@yahoo.com ORCID ID: orcid.org/0000-0001-6750-8886 Received: 29.06.2017 Accepted: 05.09.2017

${ }^{\circ}$ Copyright 2018 by Ege University Faculty of Medicine, Department of Pediatrics and Ege Children's Foundation The Journal of Pediatric Research, published by Galenos Publishing House. 
$\mathrm{fL})$. Routine biochemical analysis, including renal function parameters and serum renin level were within normal ranges. His urine was reddish and urinalysis revealed proteinuria 1+, RBC 3+.

Ultrasonography (US) revealed the left kidney with increased parenchymal echogenicity and multi septated perinephric collection. Abdominal magnetic resonance imaging on T2A weighted images revealed a multilocular hyperintense cystic lesion infiltrating renal parenchyma and surrounding the left renal cortex. The perirenal cysts were hypointense and non-contrast enhancing on $\mathrm{T} 1$ weighted images (Figure $1 A, B, C$ ). The right kidney was normal in all imaging studies. These characteristic radiological imaging findings were consistent with the appearance of left perirenal lymphangiectasia. In addition, left kidney differential function was found to be $27 \%$ by dimercaptosuccinic acid renal scintigraphy. No pathological finding was detected in renal doppler US, including left renal vein dilatation. Thus, the diagnosis of nut-cracker phenomenon was also ruled out.

The diagnosis of left perirenal lymphangiectasia was made on the basis of clinical, imaging and laboratory findings that were summarized above. The patient was managed conservatively. Angiotensin-converting enzyme (ACE) inhibitor (enalapril maleate, $0.3 \mathrm{mg} / \mathrm{kg} /$ day in 2 divided doses) was started for hypertension. On follow-up, gross hematuria was improved spontaneously and urinalysis returned to normal. After one month of enalapril therapy, his blood pressure lowered to the normal range. His hematocrit values were stable and phlebotomy was not required. The left hypo-functioning kidney did not affect global renal function. Controlled ultrasounds did not show an increase in perirenal

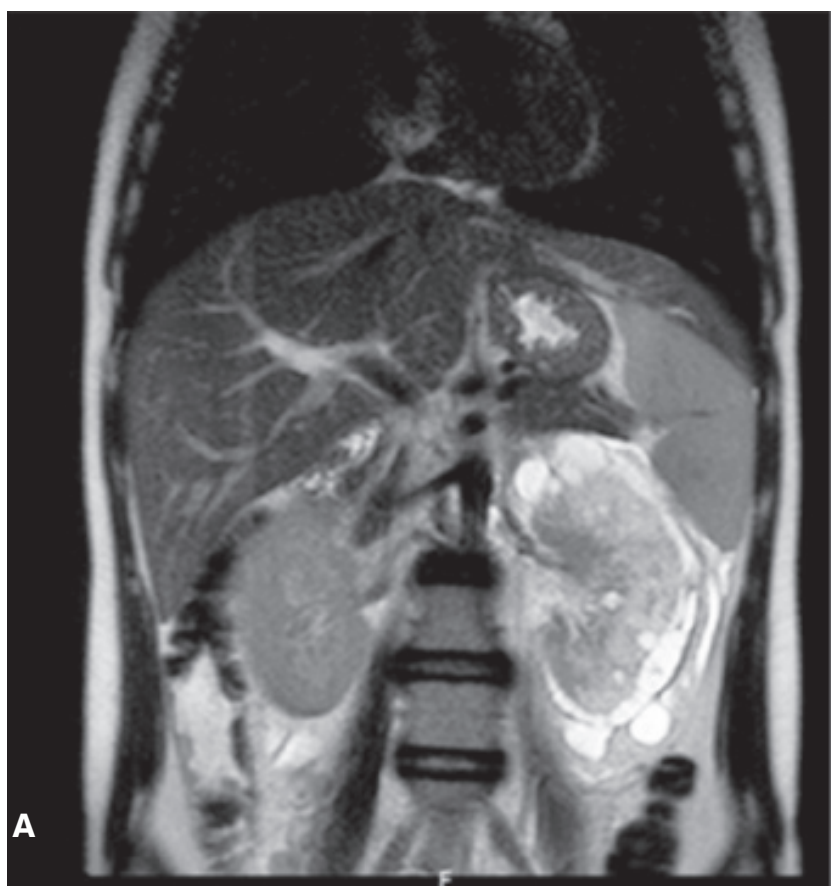

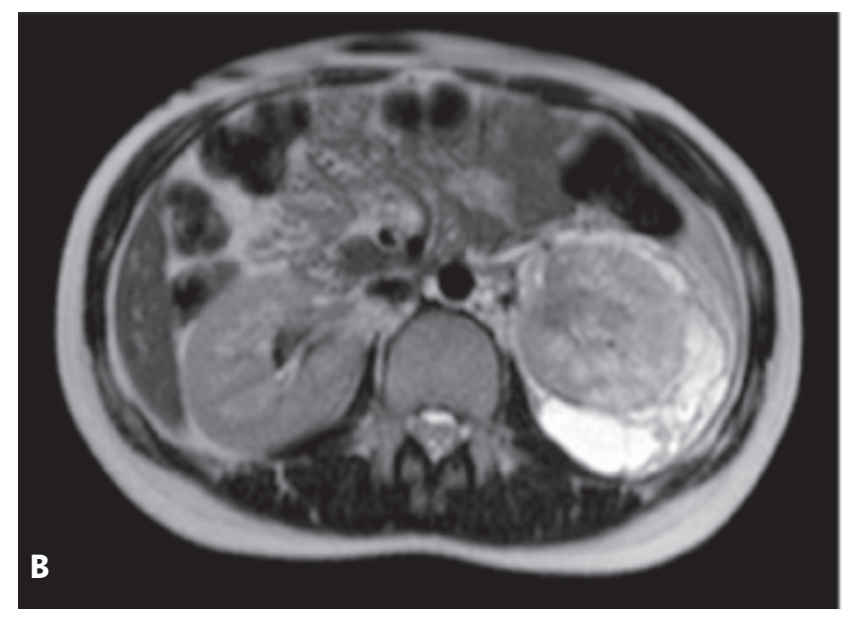

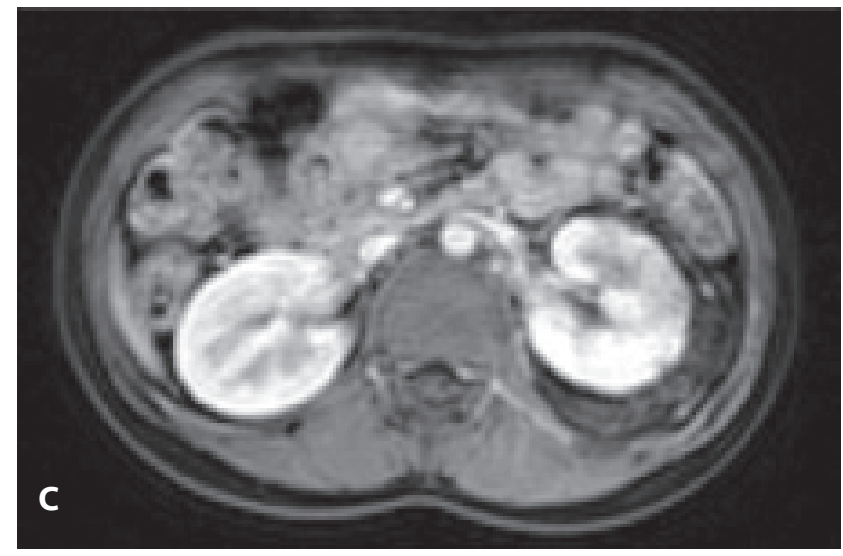

Figure 1. A) Hyperintense left perirenal cystic lesion infiltrating renal parenchyma on coronal T2 weighted magnetic resonance image, B) Hyperintense left perirenal multiple cysts on axial T2 weighted image, C) Axial post-contrast T1 fat-suppressed image showing hypointense and non-contrast enhancing left perirenal cysts

collection on the left kidney. Written informed consent was obtained from patient's parents for publication.

\section{Discussion}

In the literature, cystic RL has been more rarely described in childhood than in adulthood and it may be symptomatic in any pediatric age group including the neonatal period (7).

To our knowledge, gross hematuria as the initial manifestation of RL in childhood has been first described in our case. Similarly, although hypertension and polycythemia associated with RL are seen in adults, this association has been first reported in a pediatric case (8). The cause of both conditions is suggested to be the compression of the renal parenchyma by subcapsular collection, which results in increased erythropoietin and renin secretion due to ischaemic kidney (page kidney).

In our case, the serum renin level was normal, however, since needle aspiration of the left perirenal collection was not 
performed, we do not know the renin level in the perinephric fluid. We were also unable to detect the serum erythropoietin concentration in our center. Nevertheless, the left perinephric collection was compressing the kidney on imaging studies. Also, his high blood pressure was under control by an ACE inhibitor. Therefore, we attributed both the hypertension and polycythemia to ischaemic kidney due to compression of the left perirenal collection.

In most patients, symptoms associated with RL improve following conservative treatment as in our case. ACE inhibitors are proposed for the treatment of renin-dependent hypertension. Marsupialization or needle aspiration of the perinephric fluid can be performed for large perinephric collections. Asymptomatic patients do not require treatment.

In conclusion, RL is a rare cause of cystic renal diseases in the pediatric age group and its diagnosis is based primarily on pathognomonic imaging findings. Treatment of complications and long-term follow-up are required to reduce renal morbidity in symptomatic patients.

\section{Ethics}

Informed Consent: Written informed consent was obtained from the patient's parents.

Peer-review: Externally and internally peer-reviewed.

\section{Authorship Contributions}

Concept: Ç.S.D., Design: Ç.S.D., G.K.A., Data Collection and/or Processing: Ç.S.D., G.K.A., Literature Search: Ç.S.D., G.K.A., Writing: Ç.S.D.
Conflict of Interest: No conflict of interest was declared by the authors.

Financial Disclosure: The authors declared that this study has received no financial support.

\section{References}

1. Pandya VK, Sutariya HC, Gandhi SP, Khemchandani SI, Patel HV, Shah MK. Role of CT scan in diagnosis of renal lymphangiectasia: our single-center experience. Ren Fail 2017;39:533-9.

2. Nassiri AA, Lotfollahi L, Bakhshayeshkaram $M$, et al. Renal Lymphangiectasia: A Curious Cause of Pleural Effusion. Tanaffos 2015;14:213-6.

3. Arora R. Bilateral renal lymphangiectasia-an unusual cause of abdominal distension and flank pain in a child. Quant Imaging Med Surg 2016;6:466-9.

4. Cadnapaphornchai MA, Ford DM, Tyson RW, Lum GM. Cystic renal lymphangiectasia presenting as renal insufficiency in childhood. Pediatr Nephrol 2000;15:129-31.

5. Simonton SC, Saltzman DA, Brennom W, et al. Cystic renal lymphangiectasia: a distinctive clinicopathologic entity in the pediatric age group. Pediatr Pathol Lab Med 1997;17:293-301.

6. Ueda S, Yanagida H, Sugimoto K, et al. Chronic renal insufficiency in a boy with cystic renal lymphangiectasia: morphological findings and long-term follow-up. Clin Nephrol 2007;68:416-21.

7. Pickering SP, Fletcher BD, Bryan PJ, Abramowsky CR. Renal lymphangioma: a cause of neonatal nephromegaly. Pediatr Radiol 1984;14:445-8.

8. Blanc M, Schmutz G, BelzileF, Sabbagh R. Renal lymphangiectasia presenting with hypertension and polycythemia. Can Urol Assoc | 2014;8:163-6. 